\title{
THE NEUTRAL POINT THEORY \\ OF SOLAR FLARES
}

P. A. SWEET

University of London Observatory, England

\begin{abstract}
ABSTRAGT
It is shown that under certain conditions hydrostatic equilibrium becomes unstable in a conducting medium in the presence of a magnetic field containing a neutral point if the gas pressure is less than a limiting value.

The motion resulting from breakdown of hydrostatic equilibrium in the solar chromosphere above complex sunspot groups could produce solar flares and cosmic ray particles.
\end{abstract}

\section{INTRODUGTION}

Cowling [1] has pointed out that, if solar flares were due to Joule heating and cosmic ray particles due to electric fields in the chromosphere, electric current sheets of no more than a few metres thick would have to be set up. Dungey [2] indicated that the field near a neutral point is unstable and would constrict itself to produce current sheets of the narrowness required. This effect provided the germ of the ideas in the present paper although it is shown that the gas pressure and conditions far from the neutral point, neglected by Dungey, play essential parts in the development of the high currents.

\section{THE MEGHANIGAL FORGES DUE TO A DISTORTED FIELD WITH A NEUTRAL POINT}

Consider the fields in Fig. I, the lines of force being directed in the $x y$-plane and are independent of the $z$-coordinate. Introduce a vector potential (o, o, $A$ ), so that $A=$ constant on a line of force. It is supposed that the electric currents producing the field are situated below PQ. The two regions bounded by the lines $A=A_{2}$ in Fig. I may be regarded as the original fields due to the currents in regions $\mathrm{A}$ and $\mathrm{B}$, and the field outside as the common field resulting from the partial interpenetration of these fields. 
Suppose the current systems A and B are displaced towards each other by an appreciable fraction of their initial separation. The medium below $P Q$ is taken to be a perfect conductor, and the normal flux, and hence the value of $A$, at every point of $\mathrm{PQ}$ in each region $\mathrm{A}$ and $\mathrm{B}$ remain unaltered.

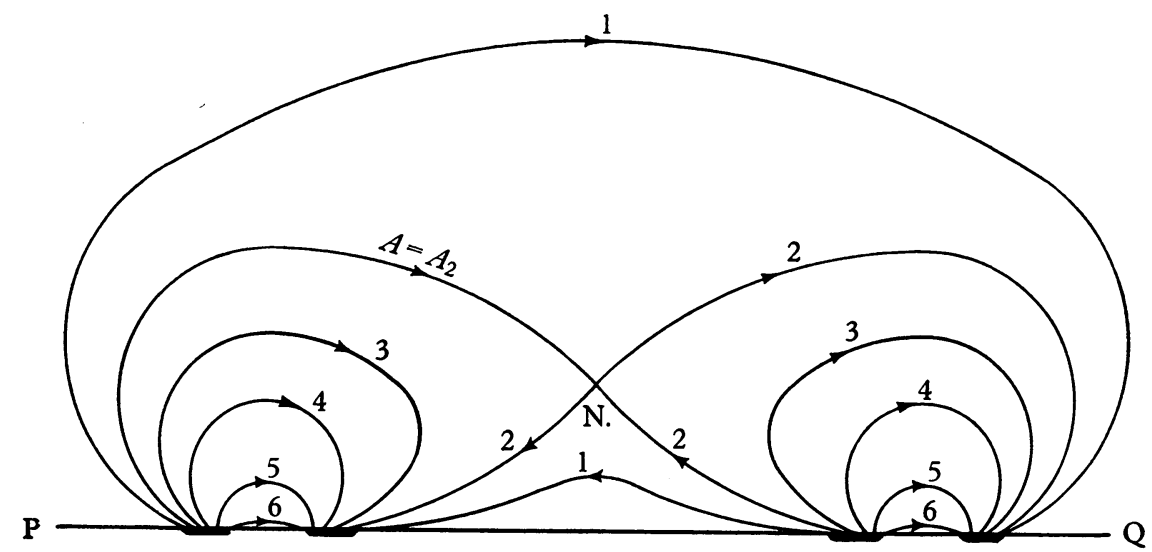

A

B

Fig. I. Potential field of two bipolar systems.

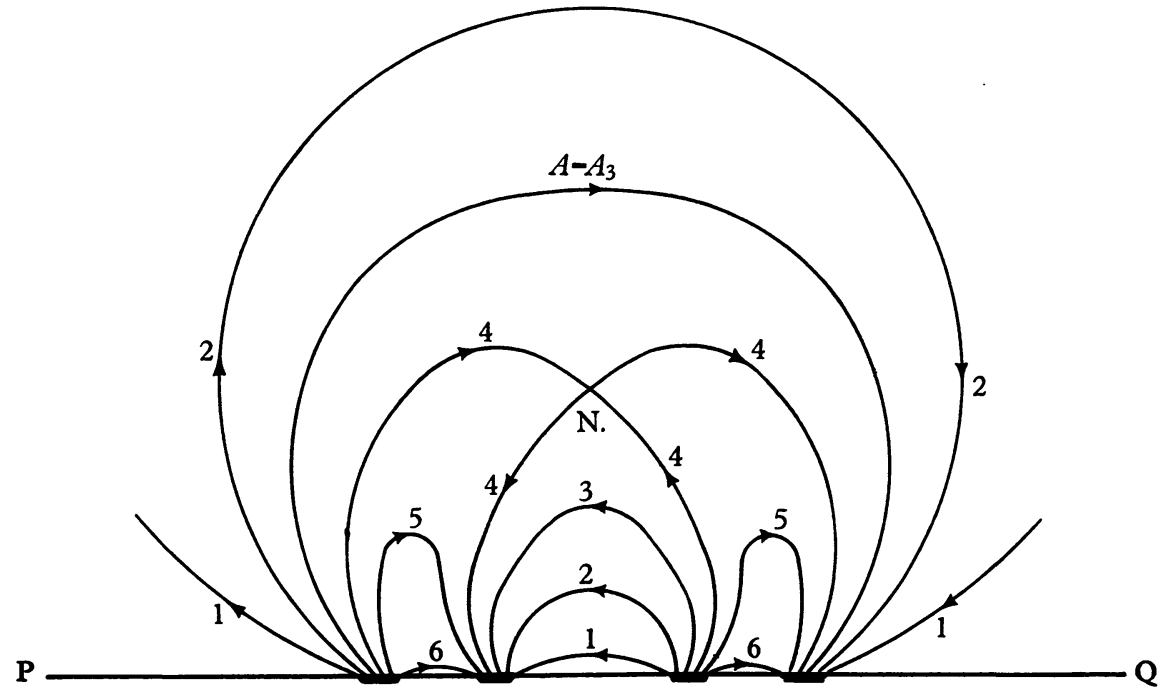

A

B

Fig. 2. Potential field of displaced systems.

If the medium above PQ were a non-conductor the two systems would suffer a further interpenetration and the value of $A$ at the neutral point would increase to values $A_{3}, A_{4}$, etc., according to the extent of the 


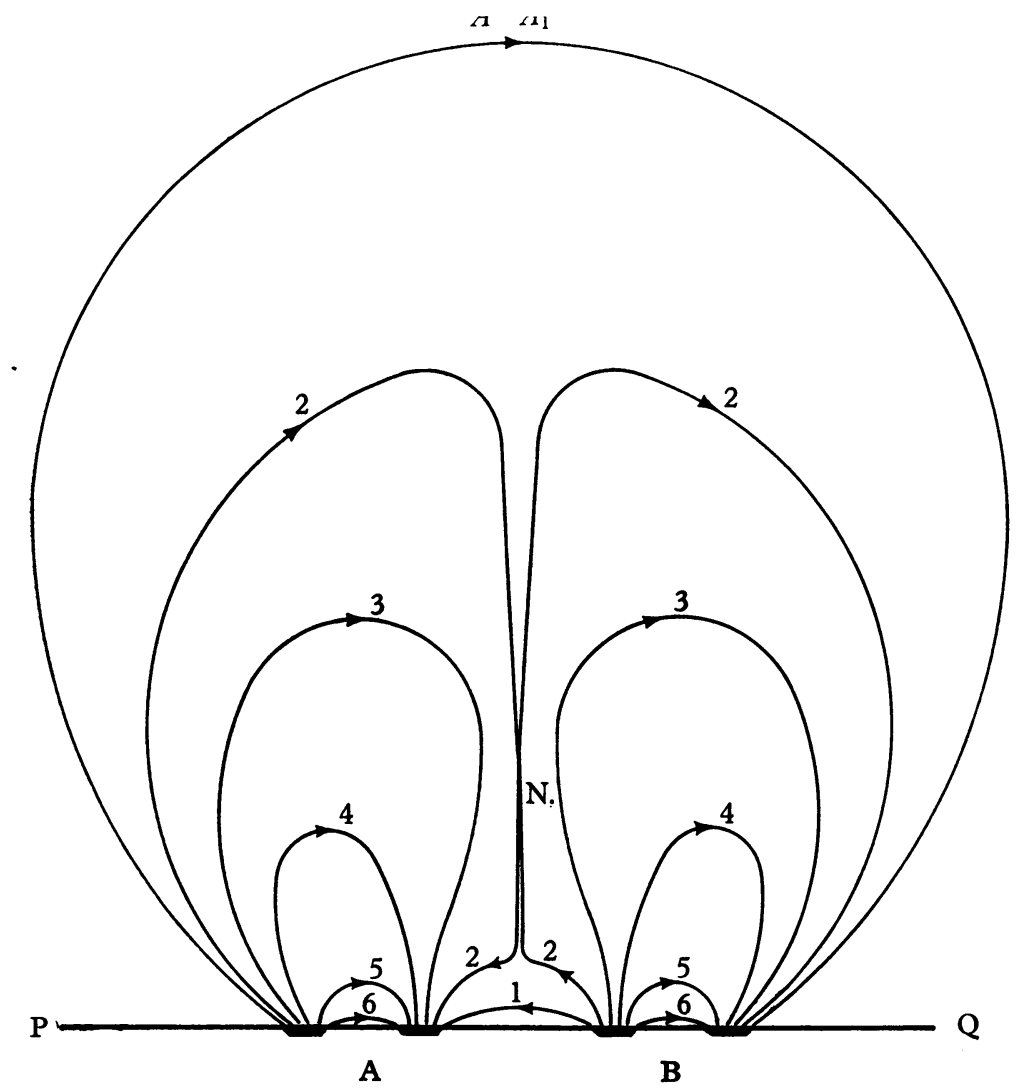

Fig. 3. Field of displaced systems in perfectly conducting medium.

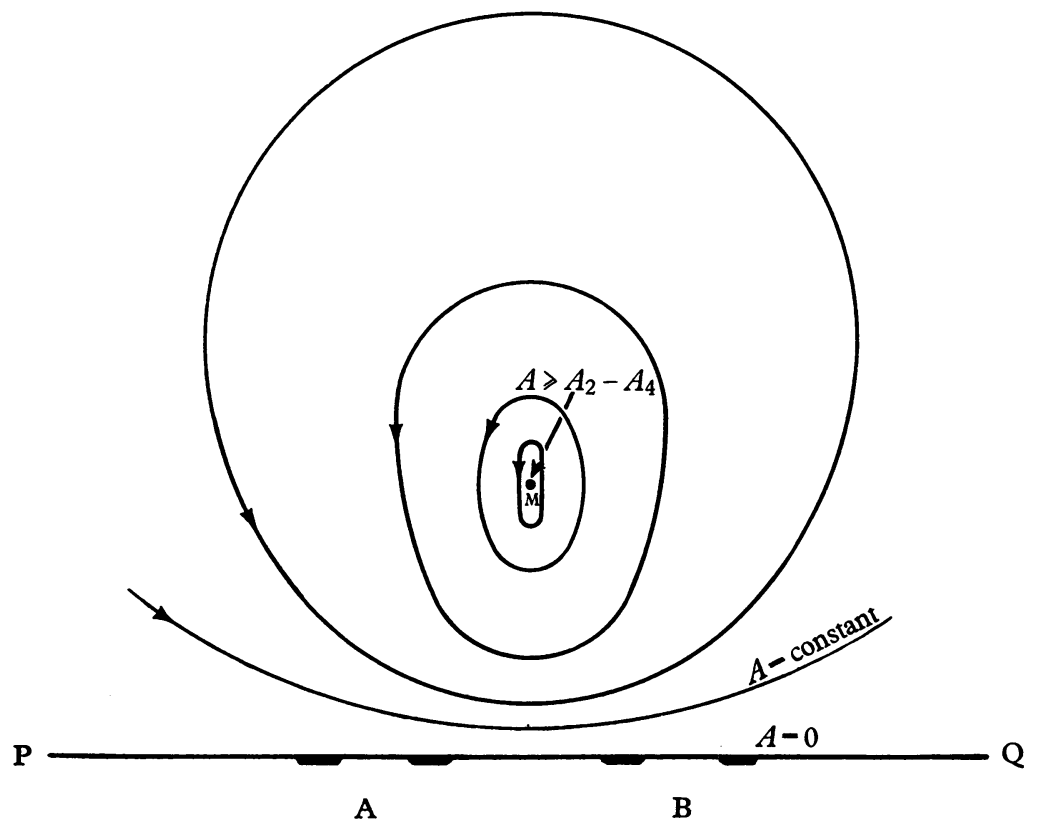

Fig. 4. The difference field. 
displacement, as in Fig. 2. No currents are induced above PQ and the field here is called the equivalent potential field.

If, on the other hand, the medium is a perfect electrical conductor the lines of force through the neutral point move with the medium and the two fields $\mathrm{A}$ and $\mathrm{B}$ do not interpenetrate further. The fields may be said to collide. The value of $A$ at the neutral point remains unaltered and the field is of the general form represented in Fig. 3. It is not unique as it depends on how the medium moves during the displacement. It has, however, the same normal components on $P Q$ as the equivalent potential field. The latter is uniquely determined by these components, hence the colliding field, which has a different value of $A$ at the neutral point, can under no circumstances be potential. The magnitude of the resulting currents will now be investigated.

Let $\left(\mathrm{o}, \mathrm{o}, A^{\prime}\right)$ and $\left(\mathrm{o}, \mathrm{o}, A^{\prime \prime}\right)$ be the vector potentials for the colliding field and the equivalent potential field, respectively, above PQ, and consider the function $A=A^{\prime}-A^{\prime \prime}$. Therefore $A=0$ on $\mathrm{PQ}$, and $\Delta^{2} A^{\prime}=\Delta^{2} A$. The lines through the neutral point of the colliding field, $A^{\prime}=A_{2}$ in Fig. 3 , connect regions $\mathrm{A}$ and $\mathrm{B}$ on $\mathrm{PQ}$. The lines through the neutral point of the equivalent potential field, $A^{\prime \prime}=A_{4}$ in Fig. 2, also connect regions $\mathrm{A}$ and $\mathrm{B}$. The latter lines intersect $P Q$ at points lying within the pairs of points of intersection of $A^{\prime}=A_{2}$ with $\mathrm{PQ}$ in each of the regions $\mathrm{A}$ and $\mathrm{B}$. The lines $A^{\prime \prime}=A_{4}, A^{\prime}=A_{2}$ must therefore intersect at least one point $\mathrm{M}$. Therefore $A_{\mathrm{M}}=A_{2}-A_{4}$. With the sense of the field as in Figs. I, 2 and $3 A_{r}$ decreases with increasing $r$, hence $A_{2}>A_{4}$ and we may write

$$
A_{\max } \geqslant A_{2}-A_{4}=\mathrm{o}\left(H_{0} L\right),
$$

where $H_{0}$ and $L$ are typical values of the field strength and linear dimensions of the field.

The analysis that follows was suggested by some of the classical work of Picard [3]. There is no loss of generality in supposing that there is only a single maximum in $A$. The system of lines $A=$ constant is then of the general form shown in Fig. 4 .

$A_{\mathrm{M}}$ may be expressed as follows:

$$
A_{\mathrm{M}}=-\frac{\mathrm{I}}{2 \pi} \iint_{1 / 2 \text {-plane }} \ln \left(r_{1} / r\right) \Delta^{2} A_{\mathrm{X}} d S,
$$

where $d S$ is an element of area of the $x y$-plane at a point $\mathrm{X}$ and $r$ and $r_{1}$ are, respectively, the distances of $X$ from $M$ and from the mirror image, $M_{1}$, of $\mathrm{M}$ in $\mathrm{PQ}$, the integration being carried out over the half-plane above $\mathrm{PQ} . \ln \left(r_{1} / r\right)$ is in fact the Green's function for this region. There are by 
definition no currents above $P Q$ other than those induced by the displacement. The principal contribution to $A_{\mathrm{M}}$ therefore arises in some region $\mathrm{D}$ whose dimensions, and in particular whose farthest point from $M_{1}$, do not exceed a value of order $L$. From (2) it is then seen that

$$
A_{\mathrm{M}}=-\frac{\lambda}{2 \pi} \iint_{\mathrm{D}} \ln \left(r_{1} / r\right) \Delta^{2} A_{\mathrm{X}} d S,
$$

where $\lambda=\mathrm{o}(\mathrm{I}) \cdot A$ is a maximum at $\mathrm{M}$, therefore $\Delta^{2} A<\mathrm{o}$ in a neighbourhood of $\mathrm{M}$; let $\mathrm{D}_{1}$ be the region within $\mathrm{D}$ formed from all points at which $\Delta^{2} A<0$. Hence

$$
A_{\mathrm{M}} \leqslant \frac{\lambda}{2 \pi} \iint_{\mathrm{D}_{\mathbf{1}}} \ln \left(r_{\mathbf{1}} / r\right)\left|\Delta^{2} A_{\mathrm{X}}\right| d S,
$$

therefore

$$
\left|\Delta^{2} A\right|_{\max } \geqslant \frac{2 \pi A_{\mathrm{M}}}{\lambda \iint_{\mathrm{D}_{1}} \ln \left(r_{1} / r\right) d S} .
$$

But the denominator on the right-hand side of $(5)$ cannot exceed a value of the order of magnitude $L^{2}$, hence, using (I), (5) shows that

$$
\left|\Delta^{2} A\right|_{\max }>\lambda_{1} H_{0} / L
$$

where $\lambda_{1}=0(\mathrm{I})$. The current density in the $z$-direction is given, in gaussian units, by $j=-c \Delta^{2} A / 4 \pi$, therefore

$$
j_{\max }>c \lambda_{1} H_{0} / 4 \pi L \text {. }
$$

The mechanical force $F_{e m}$ exerted by the current is $j H^{\prime} / c$ where $H^{\prime}$ is the field strength. If the currents exceed the limit in (7) over a region with dimensions comparable with $L$, there must be points in the region where $H^{\prime}=\mathrm{o}\left(H_{0}\right)$. Hence

$$
F_{\max }>\lambda_{2} H_{0}^{2} / 4 \pi L,
$$

where $\lambda_{2}=0(\mathrm{I})$. This inequality holds a fortiori when the currents are concentrated into a smaller region.

Consider now the hydrostatic pressure $P$ required to maintain equilibrium with the force $F_{e m}$. In hydrostatic equilibrium

$$
\operatorname{grad} P=-\Delta^{2} A^{\prime} \operatorname{grad} A^{\prime} / 4 \pi \text {. }
$$

$P$ and $\Delta^{2} A^{\prime}$ are therefore functions of $A^{\prime}$, and (9) may be integrated to give

$$
P_{\mathrm{M}}-P_{\mathrm{X}}=-\frac{\mathrm{I}}{4^{\pi}} \int_{A_{\mathrm{X}}^{\prime}}^{A_{\mathrm{M}}^{\prime}} \Delta^{2} A^{\prime} d A^{\prime}
$$

This shows that at all points $\mathrm{X}$ in a region $\mathrm{D}_{2}$ where the current exceeds the limit in (7),

$$
P_{\mathrm{M}}-P_{\mathrm{X}}>\frac{\lambda_{1} H_{0}\left(A_{\mathrm{M}}^{\prime}-A_{\mathbf{X}}^{\prime}\right)}{4^{\pi L}}
$$


If $\mathrm{D}_{2}$ has dimensions of order $L, \mathrm{X}$ can be chosen far enough from $\mathrm{M}$ so that $A_{\mathrm{M}}^{\prime}-A_{\mathrm{X}}^{\prime}=\mathrm{o}\left(H_{0} L\right)$. (I I $)$ then shows that

$$
P_{\mathrm{M}}>\lambda_{3} H_{0}^{2} / 4 \pi \text {, }
$$

where $\lambda_{3}=o(\mathrm{I})$. Alternatively, suppose $\mathrm{D}_{2}$ is narrow in one of its dimensions, corresponding to a current sheet of thickness $h \ll L$ and of width $l=\mathrm{o}(L)$. Then taking $\mathrm{D}=\mathrm{D}_{2}$ in $(3)$,

$$
A_{\mathrm{M}}>\lambda\left|\Delta^{2} A\right|_{\max } \iint_{\mathrm{D}_{\mathbf{2}}} \ln \left(r_{1} / r\right) d S / 2 \pi=\lambda_{\mathbf{4}}\left|\Delta^{2} A\right|_{\max } l h,
$$

where $\lambda_{4}=o(\mathrm{I})$. Hence

$$
\left|\Delta^{2} A\right|_{\max }=\mathrm{o}\left(A_{\mathrm{M}} / l h\right) \text {. }
$$

Let $\mathrm{X}$ be a point on the boundary of $\mathrm{D}_{2}$ near $\mathrm{M}$, then

$$
A_{\mathrm{M}}-A_{\mathrm{X}}=\mathrm{o}\left(h^{2}\left|\Delta^{2} A\right|_{\max }\right)=\mathrm{o}\left(A_{\mathrm{M}} h / l\right) \text {. }
$$

Therefore

$$
A_{\mathrm{M}}^{\prime}-A_{\mathbf{X}}^{\prime}=A_{\mathrm{M}}^{\prime \prime}-A_{\mathbf{X}}^{\prime \prime}+\mathrm{o}\left(A_{\mathrm{M}} h / l\right) \text {. }
$$

If $\mathrm{M}$ does not coincide with the neutral point of the equivalent potential field the sign of $A_{\mathrm{M}}^{\prime \prime}-A_{\mathrm{X}}^{\prime \prime}$ can be changed by taking $\mathrm{X}$ on the boundary of $\mathrm{D}_{2}$ opposite to the original point selected. A point can therefore be chosen such that

$$
A_{\mathrm{M}}^{\prime}-A_{\mathrm{X}}^{\prime}>\lambda_{5} A_{\mathrm{M}} h / l,
$$

where $\lambda_{5}=0$ (I). If $\mathrm{M}$ coincides with this neutral point then

$$
A_{\mathrm{M}}^{\prime \prime}-A_{\mathrm{X}}^{\prime \prime}=\mathrm{o}\left(h^{2} A_{\mathrm{M}} / L^{2}\right)
$$

and (I 7 ) still holds. Finally, on applying (I 7 ) and (I 4 ) to (IO),

$$
P_{\mathrm{M}}=\mathrm{o}\left(A_{\mathrm{M}}^{2} / l^{2}\right)=\mathrm{o}\left(H_{0}^{2}\right) \text {, }
$$

the same result as in (12). (12) holds a fortiori when both dimensions of the current region are small.

In all cases, therefore, $P_{\mathrm{M}}$ must exceed a value of order $H_{0}^{2}$ if hydrostatic equilibrium is to be possible without additional external forces. In applying the above criteria it must be remembered that the forces immediately below PQ required to force the current systems A and B together are themselves of order $H_{0}^{2} / L$. In a continuous system, therefore, the forces immediately above PQ may also be of this order of magnitude. The criteria would apply, however, whenever the scale height of the forces below PQ is small compared with $L$. This certainly obtains in the case of sunspot fields. For spot fields the gravitational forces and the gas pressures in the chromosphere fall far short of the limits in (8) and (12). Hydrostatic equilibrium would therefore break down over a complex spot group in which, along a line of 
minimum resultant field strength, the field was much smaller than the typical value, ${ }^{*}$ and in which the components of the group were moving relative to each other.

\section{PHYSICAL INTERPRETATION OF THE EQUILIBRIUM GRITERIA}

As the displacements in the photosphere proceed the magnetic field transmits the sub-photospheric force to the chromosphere. The resulting distortion in the chromospheric field will be greatest where the field is weakest, i.e. near the neutral line. The field there will tend to flatten, the effect

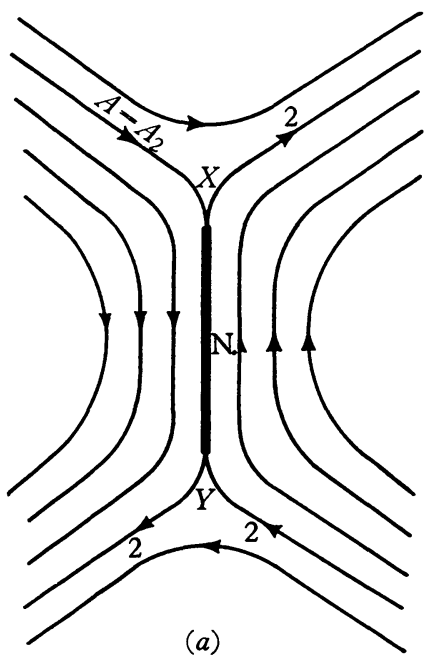

Fig. 5. The collision layer. (a) Field in neighbourhood of current sheet. (b) Field across current sheet. (c) Idealized hydrodynamic model.

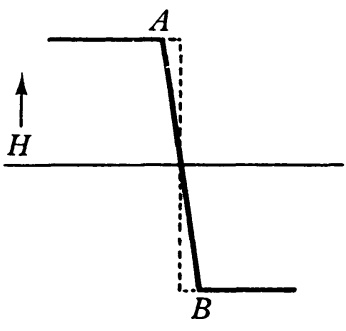

(b)

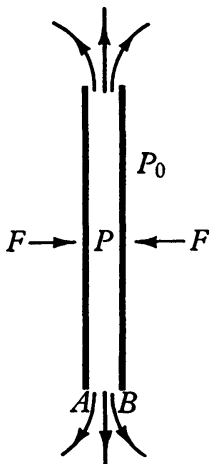

(c)

being analogous to the flattening of a motor tyre when loaded. A limit is reached when hydrostatic equilibrium breaks down. A thin collision layer of gas is then formed as in Figs. $5(a)$ and $(b)$. There is an inward magnetic pressure at the boundaries approximately equal to $H_{1}^{2} / 8 \pi$ where $H_{1}$ is the field strength there. This is uncompensated along the middle line of the layer where the field strength is small. Hydrostatic equilibrium then requires a gas pressure of approximately $H_{1}^{2} / 8 \pi$ in the middle of the layer, and since the layer is effectively open at its ends this means that the gas pressure surrounding the layer must be of the same order of magnitude. For large sub-photospheric displacements $H_{1}=\mathrm{o}\left(H_{0}\right)$, and the criterion in (12) follows.

* Such lines are generalizations of the neutral lines in the present two-dimensional analysis. 
4. THE DYNAMIGS OF THE COLLISION LAYER

An exact treatment of the motion is not considered in the present paper. Instead, an analogy in pure hydrodynamics is considered. Consider two parallel rigid plates, as illustrated in Fig. $5(c)$, with infinite extension in the $z$-direction. Let the distance $h$ between the plates be small compared with their length $2 l$. The region between the plates is occupied by gas and the surrounding gas pressure is taken to be negligible. Suppose the plates are being forced together by a constant force $F$ per unit $z$-length. The gas offers little resistance to the plates until sufficiently compressed. After this a quasi-steady state is set up in which gas flows out from the ends of the layer and the plates approach each other with a velocity that is small compared with the gas velocities along the layer. Take axes centred at the mid-point of one plate with the $x$-axis along the plate and the $y$-axis directed toward the other plate. Let the gas velocity be $(u, v, o)$ and the downward velocity of the upper plate be $V$. The flow is nearly steady and is almost one-dimensional so that the pressure $P$, the density $\rho$ and the $x$-component of the velocity are nearly functions of $x$ only. The $y$-component of the velocity is given by

$$
v=-V y / h \text {. }
$$

The equation of continuity $\operatorname{div} \rho \mathbf{v}=0$ reduces to

$$
u \frac{d \ln \rho}{d x}+\frac{d u}{d x}=\frac{V}{h} \text {. }
$$

The motion is irrotational, hence Bernoulli's equation

$$
u^{2}=2 \int_{P}^{P_{0}} \frac{d P}{\rho}
$$

holds, where the suffix o denotes values at the origin. For simplicity in the analysis isothermal flow is assumed. The results for isentropic flow do not differ radically from the isothermal case, so that in the circumstances the extra analysis involved in the isentropic case is not justified. A fuller analysis would in any case have to take into account the effects of radiative cooling and Joule heating. In flare conditions the rate of radiative cooling is sensitive to the temperature. This would keep the temperature low in spite of heavy Joule heating. Thus take

$$
\frac{P}{\rho}=\frac{P_{0}}{\rho_{0}}=\frac{\Re T}{\mu},
$$

where $T$ is the temperature, $\mathfrak{R}$ is the gas constant and $\mu$ is the mean mole- 
cular weight of the gas in units of the mass of the hydrogen atom. On solving (20)-(23) $u$ is given by $u=q \xi$, where $q^{2}=\Re T / \mu$ and

$$
\xi\left(\mathrm{I}-\frac{1}{3} \xi^{2}\right)=V x / q h \text {. }
$$

$P$ and $\rho$ are given by $\quad \rho / \rho_{0}=P / P_{0}=e^{-\frac{1}{2} \xi^{2}}$.

The boundary condition at the ends of the layer, $x= \pm \mathrm{I}$, is determined by the external pressure. As this is assumed to be negligible compared with $P_{0}$ it is therefore below the so-called critical pressure for outflow from a tube (see for example Prandtl [4]). In the isothermal case this pressure is $P_{0} / \sqrt{ } e$; the pressure at the ends is equal to this, therefore (25) shows that $\xi=\mathrm{I}$ at $x=\mathrm{I}$. On integrating along the plates,

$$
F=2 \int_{0}^{1} P d x
$$

When reduced by means of the previous equations, this shows that

Again, on putting $x=\mathrm{I}$ in (24),

$$
F=\frac{2 q}{\sqrt{ } e} \frac{P h}{V}
$$

$$
V=\frac{2 q}{3} \frac{h}{l}
$$

therefore

$$
P_{0}=\frac{\sqrt{ }(e) F}{3 l} .
$$

Equations (24), (25), (28) and (29) completely describe conditions in a layer of given length and thickness. The subsequent behaviour of the layer is considered by remembering that $d h / d t=-V$, hence from (28),

$$
d h / d t=-2 q h / 3 l \text {. }
$$

Further progress cannot be made until the behaviour of the length of the layer is known. This depends on the rate of dissipation of magnetic energy by Joule heating in the layer.

\section{THE ELEGTROMAGNETIGS OF THE GOLLISION LAYER}

The problem will again be simplified by adopting Ohm's law in a medium moving with velocity $(u, v, 0)$ :

$$
j / \sigma=E+\left(v H_{x}-u H_{y}\right) / c,
$$

where $\sigma$ e.s.u. is the conductivity and $E$ is the electric intensity in the $z$-direction. This neglects the effect of Hall current, but the reduction in 
conductivity due to collisions with neutral atoms as derived by Cowling [5] and by Piddington [6] could be allowed for by adopting the appropriate value of $\sigma$ once more was known about conditions in the layer.

Maxwell's equation curl $\mathbf{E}=-\frac{\mathbf{I}}{c} \frac{\partial \mathbf{H}}{\partial t}$ can be integrated and written

$$
E=-\frac{1}{c} \frac{\partial A}{\partial t}+f(t)
$$

where $f(t)$ is a function to be determined. By eliminating $E$ between (3I) and (32) and using the relation $j=-c \Delta^{2} A / 4 \pi$,

$$
\frac{\partial A}{\partial t}=-\frac{c^{2}}{4 \pi \sigma} \Delta^{2} A+c f(t)+\left(v H_{x}-u H_{y}\right) .
$$

In the photosphere $A$ is independent of time by definition, there is no fluid velocity, and $\Delta^{2} A / \sigma$ is supposed negligible. (33) therefore shows that $f(t)=0$. At the neutral point $\mathrm{N} H=0$ and (33) shows that

$$
d A_{\mathrm{N}} / d t=-c^{2}\left[\Delta^{2} A\right]_{\mathrm{N}} / 4 \pi \sigma=-\lambda_{6} c^{2} a_{\mathrm{N}} / 4 \pi \sigma h l,
$$

where $\lambda_{6}$ is a dimensionless quantity of order unity.

The value of $A_{\mathrm{N}}$ effectively determines the length of the layer.

It is not possible to derive the precise relation on the simple analogy of the previous section. An empirical relation is therefore introduced in the form

$$
A_{\mathrm{N}}=A_{\mathrm{N}}(\mathrm{o})\left(l / l_{0}\right)^{n} \text {, }
$$

where $n$ is a positive constant and $A_{\mathrm{N}}(0)$ and $l_{0}$ are values at a given epoch $t=0$ soon after the quasi-steady state is attained. (34) then shows that

$$
\frac{d l}{d t}=-\frac{c^{2}}{4 \pi \sigma n h}
$$

taking $\lambda_{8}=\mathrm{I}$ for definiteness. On dividing (36) and (30) and integrating,

$$
l=l_{0} \exp \left\{h_{\mathbf{1}}\left(\mathrm{I} / h_{0}-\mathrm{I} / h\right)\right\},
$$

where $h_{0}$ is the initial value of $h$ and

On substituting (37) into (30),

$$
h_{1}=\frac{3 c^{2}}{8 \pi \sigma n q} \text {. }
$$

$$
\frac{d h}{d t}=-\frac{2 q h}{3 l_{0}} \exp \left\{h_{1}\left(\mathrm{I} / h-\mathrm{I} / h_{0}\right)\right\} .
$$

Thus $h$ may be derived implicitly at any time from

$$
t=\frac{3 l_{0}}{2 q} e^{h_{1} / h_{0}} \int_{h_{1} / h_{0}}^{h_{1} / h} \frac{e^{-x} d x}{x} .
$$

132 
The layer vanishes after a time

$$
\frac{3 l_{0}}{2 q} e^{h_{1} / h_{0}} \int_{h_{1} / h_{0}}^{\infty} \frac{e^{-x} d x}{x} .
$$

The electric intensity at the neutral point is given by

$$
E_{\mathrm{N}}=-\frac{\mathrm{I}}{c} \frac{d A_{\mathrm{N}}}{d t} \sim \frac{c A_{\mathrm{N}}(\mathrm{o}) l^{n-1}}{4^{\pi \sigma h l_{0}^{n}}} \sim \frac{c H_{0}}{4^{\pi \sigma h}} \exp \left\{(n-\mathrm{I}) h_{1}\left(\mathrm{I} / h_{0}-\mathrm{I} / h\right)\right\}
$$

taking $A_{\mathrm{N}}(\mathrm{o})=\mathrm{o}\left(H_{0} l_{0}\right)$.

The rate of Joule heating is $\sigma E_{\mathrm{N}}^{2}$. If this is taken as typical of the whole layer, then the total rate of heating/ $/ \mathrm{cm}$ of the layer in the $z$-direction is given by

$$
J \sim \sigma E_{\mathrm{N}}^{2} h l=\frac{c^{2} H_{0}^{2} l_{0}}{\mathrm{I} 6 \pi^{2} \sigma h} \exp \left\{(2 n-\mathrm{I}) h_{1}\left(\mathrm{I} / h_{0}-\mathrm{I} / h\right)\right\} .
$$

If $2 n \leqslant \mathrm{I}$ then $J \rightarrow \infty$ as $h \rightarrow 0$. We therefore take $2 n>\mathrm{I}$, in which case $J$ has a maximum value given by

$$
J_{\max } \sim \frac{H_{0}^{2} l_{0} n q}{6 \pi(2 n-\mathrm{I})} \exp \left\{(2 n-\mathrm{I}) h_{1} / h_{0}-\mathrm{I}\right\}
$$

where

$$
h=(2 n-\mathrm{I}) h_{1} \text {. }
$$

This maximum occurs at time

$$
t_{1}=\frac{3 l_{0}}{2 q} e^{h_{1} / h_{0}} \int_{h_{1} / h_{0}}^{1 /(2 n-1)} \frac{e^{-x} d x}{x},
$$

and the time from maximum activity until the layer vanishes is given by

$$
t_{2}=\frac{3 l_{0}}{2 q} e^{h_{1} / h_{0}} \int_{1 /(2 n-1)}^{\infty} \frac{e^{-x} d x}{x} .
$$

The electric intensity, in the $z$-direction, at maximum activity, is given by

$$
E_{\max } \sim \frac{2 n q H_{0}}{3(2 n-\mathrm{I}) c} \exp \left\{(n-\mathrm{I}) h_{1} / h_{0}-(n-\mathrm{I}) /(2 n-\mathrm{I})\right\} \text {. }
$$

\section{APPLICATION TO A SOLAR FLARE}

Consider a complex sunspot group with a neutral point near the base of the chromosphere, and suppose that the components of the group suffer relative displacements that break down equilibrium and produce a collision layer of length $l_{0}=10^{9} \mathrm{~cm}$. Take $T=10^{4}{ }^{\circ} \mathrm{K}$ and $H_{0}=10^{3}$ gauss as further typical values. The effective value of $\sigma$, if collisions with neutral atoms are taken into account as in the work of Cowling [5] and Piddington [6], 
would be of the order of magnitude $10^{8}$ e.s.u. It will be seen that the precise value has a negligible effect on both the rate of heating and the electric field. The results are not sensitive, in order of magnitude, to $n$ provided this is not too near I/2. Take $n=\mathrm{I}$ for definiteness, and suppose that the flare operates along a line of length $10^{10} \mathrm{~cm}$ in the $z$-direction.

With the above values, $h_{1}=1 \cdot 2 \times 10^{6} \mathrm{~cm}$. The quasi-steady state sets in as soon as the layer becomes thin compared with its length. Hence, taking $h_{0}=10^{8} \mathrm{~cm}, h_{1} / h_{0} \ll \mathrm{I}$. The maximum rate of Joule heating in the whole flare is then practically independent of $\sigma$, and is given by

$$
J_{\max }=\mathrm{I} \cdot 5 \times 1 \mathrm{I}^{29} \mathrm{ergs} / \mathrm{sec} \text {. }
$$

The time of duration of the flare at maximum activity is given by $t \sim \mathrm{IO}^{4}$ seconds, and the total energy output from the flare is therefore of order $\mathrm{IO}^{33} \mathrm{ergs}$. The electric intensity at maximum activity is given by

$$
E_{\max }=6 \text { volts } / \mathrm{cm} \text {; }
$$

this value, also, does not depend on $\sigma$. The corresponding difference in potential between the ends of the flare is $6 \times 10^{10}$ volts.

The above figures agree with the values associated with a large flare, to within the uncertainties of the theory.

\section{GONGLUSIONS}

The theory just developed reproduces some important features of solar flares. It gives a total radiation of energy and a duration of the right order of magnitude, and can account for the production of high-energy particles. It has been demonstrated conclusively that hydrostatic equilibrium can break down along a neutral line, and a narrow colliding layer set up in the ensuing motion. The theory cannot be accepted as definitive, however, until the hydrodynamic analogy introduced is replaced by a proper analysis of the dynamics of the colliding layer.

\section{REFERENGES}

[1] Cowling, T. G. The Sun, ed. G. P. Kuiper (Chicago, I953), ch. 8.

[2] Dungey, J. W. Phil. Mag. 44, 725, 1953.

[3] Picard, E. Cahiers Scientifiques, 5, 1930, ch. 8.

[4] Prandtl, L. Essentials of Fluid Dynamics (Blackie, London, 1952), p. 266.

[5] Cowling, T. G. Mon. Not. R. Astr. Soc. 116, I I 4, 1956

[6] Piddington, J. H. Mon. Not. R. Astr. Soc. ri4, 638 and 651, 1955. 